\title{
Relaxation Mechanisms of the Photoelectrons in the Second Miniband of a Superlattice Structure
}

\author{
Chun-Chi Chen, H. C. Chen, Mao-Chieh Hsu, Wen-Hsing Hsieh, Chieh-Hsiung Kuan, Member, IEEE, \\ Shiang-Yu Wang, and Chien-Ping Lee, Fellow, IEEE
}

\begin{abstract}
Photoelectron relaxation in the second miniband of a superlattice was studied with a high-pass energy filter at one end of the superlattice. By analyzing the spectral responses with different biases across the energy filter, the photoelectron distribution and relaxation in the second miniband of the superlattice can be resolved. In this paper, a model based on the rate equation is proposed to extract the related parameters of the photoelectron transport. The photoelectrons suffer from both intraminiband and interminiband relaxation. The extracted interminiband relaxation time is $11 \mathrm{ps}$, while the intraminiband one is in the subpicosecond range. In addition, this structure can also be utilized as an infrared photodetector. Our analysis shows that the fast intraminiband relaxation is the dominant mechanism of the maximum achievable responsivity of the detector and the theoretical model not only provides the insight of the photoelectron transport in the superlattice miniband but also is useful for designing a superlattice infrared photodetector.
\end{abstract}

Index Terms-Infrared photodetector, intersubband, quantumwell infrared photodetector, superlattice.

\section{INTRODUCTION}

$\mathbf{T}$ HE BENEFITS of using intersubband infrared photodetectors for large imaging system have driven studies on electron transport in the popular adopted quantum-well (QW) structures for optimization of performances. One of the methods to analyze the electron transport is using optically stimulated hot-electron spectroscopy (OSHES) [1]. In this method, an energy filter is designed to separate photoelectrons with different energies. Therefore, the energy distribution of the photoelectrons can be resolved.

In addition to QWs, superlattices are another promising structure for infrared detection and has drawn much attention. Superlattice with a graded barrier was fabricated for photovoltaic detection in the wavelength ranges of 3.6-6.3 and 8-10.5 $\mu \mathrm{m}$ in 1988 [2] and 1990 [3], respectively. In 1991 [4], superlattice alone was applied in the detection for 5-10- $\mu \mathrm{m}$ wavelength range. Superlattice with a blocking layer for low bias operation was demonstrated in 1992 [5]. The earlier works of the super-

Manuscript received July 10, 2002; revised October 21,2002. This project is supported by National Science Council of Taiwan under Contract NSC 89-2215-E-002-058.

C.-C. Chen, H. C. Chen, M.-C. Hsu, W.-H. Hsieh, and C.-H. Kuan are with the Department of Electrical Engineering and, Graduate Institute of Electronics Engineering, National Taiwan University, Taipei 10617, Taiwan, R.O.C. (e-mail: kuan@cc.ee.ntu.edu.tw).

S.-Y. Wang is with the Institute of Astronomy and Astrophysics Academia Sinica, Taipei 10617, Taiwan, R.O.C.

C.-P. Lee is with the Department of Electronic Engineering, National Chiao

Tung University, Hsinchu 30050, Taiwan, R.O.C.

Digital Object Identifier 10.1109/JQE.2002.807175 lattice-based infrared photodetectors point out the advantages of using superlattices, which include broader absorption spectrum, lower operating voltage, more suitable for low background and low operating temperature conditions [6] than the conventional QWIP. However, experimental and theoretical studies about the transport and relaxation of the photoelectrons within the miniband of the superlattice do not attract much attention.

In order to study the photoelectron transport and relaxation within the superlattice miniband, a structure that is composed of a 14-period GaAs- $\mathrm{Al}_{0.27} \mathrm{Ga}_{0.73}$ As superlattice and a high-pass energy filter (similar to the of OSHES method) is studied in this paper. In addition, this structure is also applicable as an infrared photodetector for $\sim 10 \mu \mathrm{m}$ [7]. Because of the energy filter, the photoresponse is tunable by external bias to enable multiwavelength operation and is therefore useful in versatile applications, including target discrimination and temperature sensing. Meanwhile, according to the measured voltage-dependent photoresponses, a model based on the rate equations that involves with the absorption, transport and relaxation of the electrons in the superlattice is proposed.

This paper is organized as follows. Section I is a brief introduction. The structure and measured photoresponses are presented in Sections II and III, respectively. In Section IV, a model is suggested to analyze the photoelectron transport from the observed voltage-dependent photoresponses. In the analysis, it is found that the photoelectrons in the second miniband suffer not only interminiband but also intraminiband relaxation. The photoelectrons relax back into the first miniband through interminiband relaxation, while the intraminiband one causes them to accumulate in the bottom states of the second miniband. The latter process is much faster than the former and poses a limitation on the short-wavelength responsivity. Section V summarizes this paper.

\section{SAMPLE STRUCTURE}

The schematic band diagram of the structure for analyzing the photoelectron transport in the superlattice miniband is shown in Fig. 1. The structure, grown by molecular beam epitaxy on a semi-insulating GaAs substrate, contains a 500-nm heavily doped $\mathrm{GaAs}$ bottom contact layer, a $150-\mathrm{nm} \mathrm{Al}_{0.23} \mathrm{Ga}_{0.77} \mathrm{As}$ high-pass energy filter, a 14-period GaAs- $\mathrm{Al}_{0.27} \mathrm{Ga}_{0.73} \mathrm{As}$ superlattice, and a 400-nm heavily doped top contact layer. Contact layers are both doped with $1 \times 10^{18} \mathrm{~cm}^{-3}$ of Si. Each period of the superlattice consists of $6.5 \mathrm{~nm} \mathrm{GaAs}$ well doped with $5 \times 10^{17} \mathrm{~cm}^{-3}$ of $\mathrm{Si}$ and 3.5-nm undoped $\mathrm{Al}_{0.27} \mathrm{Ga}_{0.73} \mathrm{As}$ barrier. The shadowed region in Fig. 1 shows the two minibands formed in the superlattice structure. Each miniband con- 


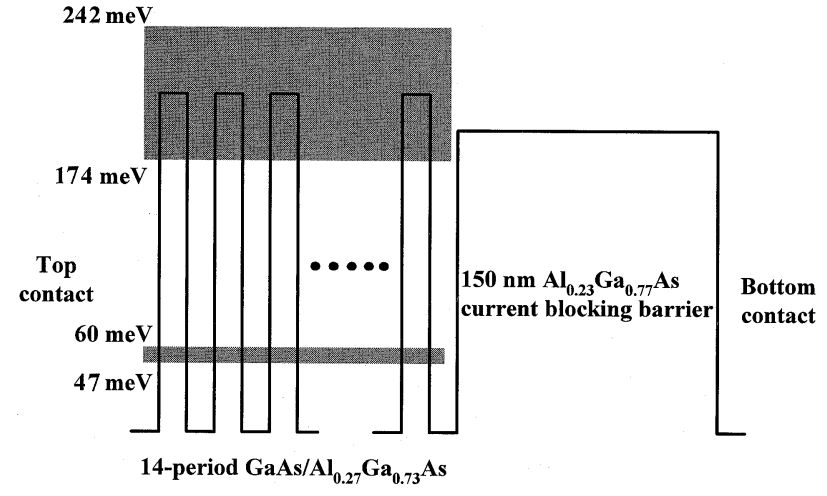

Fig. 1. Schematic band diagram of the superlattice infrared photodetector. The superlattice is composed of 6.5-nm GaAs well and 3.5-nm $\mathrm{Al}_{0.27} \mathrm{Ga}_{0.73} \mathrm{As}$ barrier. The $150 \mathrm{~nm} \mathrm{Al}_{0.23} \mathrm{Ga}_{0.77} \mathrm{As}$ current blocking layer and the superlattice barriers are both undoped and the GaAs well region of the superlattice is doped with $5 \times 10^{17} \mathrm{~cm}^{-3}$ of Si. From the conduction band edge of the GaAs, two minibands are formed in the superlattice region that ranges from 47 to $60 \mathrm{meV}$ and 174 to $242 \mathrm{meV}$, respectively.

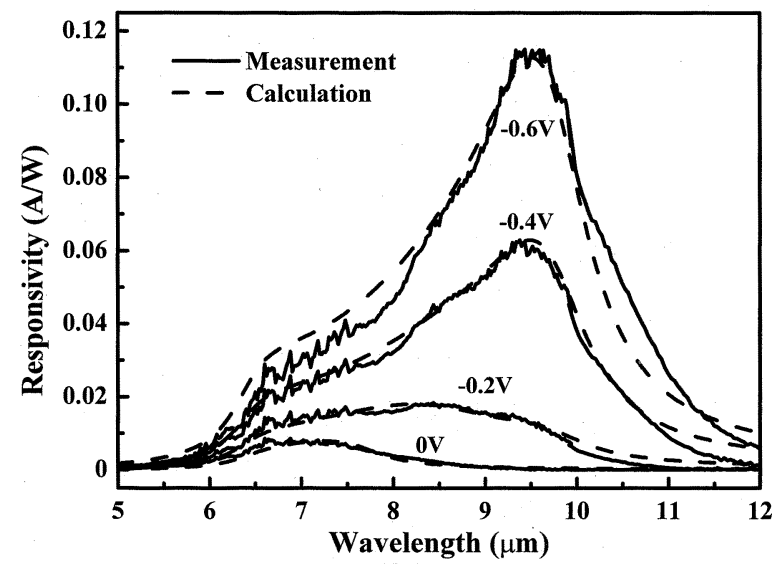

Fig. 2. Spectral responsivity from 0 to $-0.6 \mathrm{~V}$ at various biases at $25 \mathrm{~K}$. The solid curves are the measured data and the dashed curves are the simulated ones. The responsivity at $9.7 \mu \mathrm{m}$ is suppressed at zero bias, while it increases with applied bias and dominates the spectral responsivity at $-0.6 \mathrm{~V}$.

tains 14 resonant-tunneling states. The conduction band offset of $\mathrm{Al}_{x} \mathrm{Ga}_{1-x} \mathrm{As}$ relative to GaAs is taken as $810 \mathrm{x}$ meV [8]. Relative to the conduction band edge of GaAs, the first miniband ranges from 47 to $60 \mathrm{meV}$ and the second miniband ranges from 174 to $242 \mathrm{meV}$.

The barrier height $(186 \mathrm{meV})$ of the energy filter is designed to be higher than the bottom state of the second miniband in the superlattice. At around zero bias, photoelectrons with energy higher than the energy filter can pass through it, while those with energy lower are blocked. At high biases, due to the assistance of the strong electric field on the blocking barrier, part of the blocked photoelectrons can tunnel through the current blocking barrier and contribute to the photocurrent. As a result, the energy filter can be used to distinguish the energy of the photoelectron and resolve the photoelectron distribution versus energy.

The sample was fabricated into a $200 \times 400 \mu \mathrm{m}^{2}$ mesa by standard photolithography, chemical wet etching, evaporation and lift-off processes. The top and bottom contacts were made by evaporating 100-nm Ni-Ge-Au and 200-nm Au. After evaporation and lift-off, thermal annealing at $390^{\circ} \mathrm{C}$ was performed

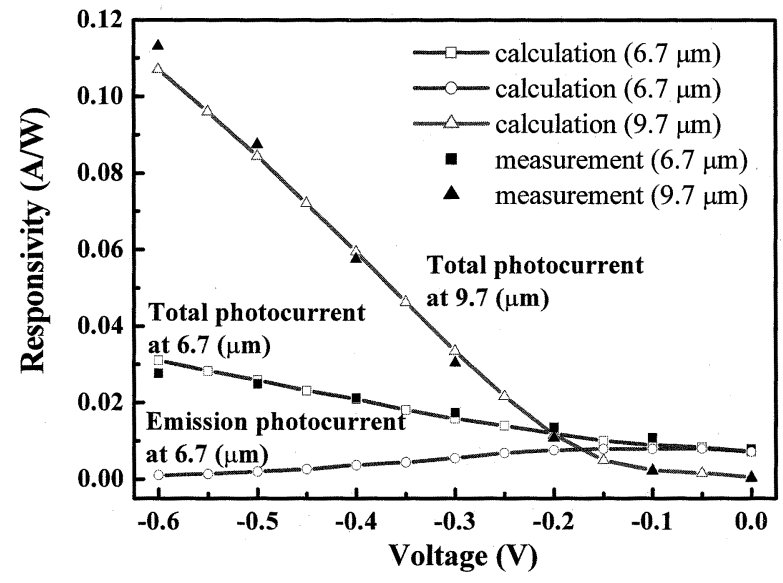

Fig. 3. Calculated and measured responsivity versus bias at 6.7 and $9.7 \mu \mathrm{m}$. The solid (open) squares and triangles represent the measured (calculated) responsivity at 6.7 and $9.7 \mu \mathrm{m}$, respectively. The open circles represent the responsivity at $6.7 \mu \mathrm{m}$ calculated by counting only the photoelectrons with energy higher than the energy filter.

to make ohmic contacts with the $n^{+}$contact layers in the semiconductor. Finally, a $45^{\circ}$ facet on the substrate was polished to allow the detector to probe the TM polarized infrared radiation.

\section{MEASURED SPECTRAL RESPONSIVITY}

The spectral photoresponses were measured by a lock-in amplifier at various temperatures and biases. A glowbar radiator was used as the light source and the light wavelength was selected with a monochromator. The peak responsivity was calibrated by a blackbody radiation source heated at $500{ }^{\circ} \mathrm{C}$. The observed spectral photoresponses show little temperature dependence and the representative one at $25 \mathrm{~K}$ is shown in Fig. 2. It is obvious that at zero bias, the detected wavelength is primarily at $6.7 \mu \mathrm{m}$. While negative bias increases in magnitude, another peak at $9.7 \mu \mathrm{m}$ rises and dominates the spectral responsivity at high bias region. Therefore, the spectral photoresponse is tunable by the external bias between 6.7 and $9.7 \mu \mathrm{m}$, which allows the structure to be applicable in versatile applications including temperature sensing and target discrimination. This experimental result agrees with the aforementioned design principle.

The measured voltage dependent responsivity at 6.7 and $9.7 \mu \mathrm{m}$ are shown as the solid squares and triangles in Fig. 3, respectively. The nonzero $6.7-\mu \mathrm{m}$ photoresponse at zero bias indicates the photocurrent is caused by the emission of the photoelectrons with energy higher than the energy filter. It is also noted that the $6.7-\mu \mathrm{m}$ photoresponse does not saturate with the bias magnitude, but increases with it instead. This is attributed to the tunneling of the relaxed photoelectrons accumulating in the bottom state of the second miniband through the energy filter by the electric field.

These voltage dependences of the photoresponse show clues for the photoelectron transport in the second miniband of the superlattice. In the next section, a model for the photoelectron transport is suggested to explain the measured voltage-dependent photoresponse. 


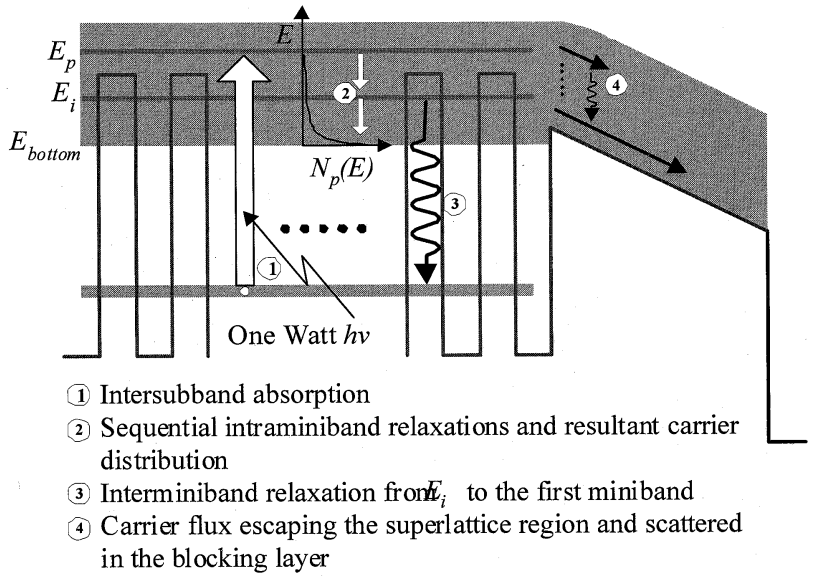

Fig. 4. Schematic drawing of the photoelectron relaxation processes in the second miniband.

\section{MODEL FOR THE RESPONSIVITY}

As is schematically drawn in Fig. 4, photons with energy $\hbar \omega$ excite electrons from the first miniband into an excited state with energy $E_{p}$ in the second miniband. The number of excited photoelectrons that move toward or backward the energy filter are equal in probability. During transport, two primary scattering processes for the photoelectrons are expected to be phonon and impurity scattering. According to the conservation of energy and crystal momentum, the electron scattering with a phonon emitted in a bulk semiconductor is allowed only in the forward direction [9]. Since the structure under investigation is operated at low temperatures $(<55 \mathrm{~K})$, phonon emission is the dominant process. Therefore, the phononrelated scattering of photoelectrons in the superlattice structure is expected to be in the forward direction (scattering angle $<90^{\circ}$ ). The electron scattering by impurities in a bulk semiconductor is inversely proportional to the forth power of the sine of the scattering angle in the case of negligible screening [10]. Therefore, the scattering of photoelectrons in the superlattice miniband by impurities is also expected to be in the forward direction. The forward-scattering property of the photoelectrons simplifies our analysis by decoupling the rate equations related with the respective numbers of photoelectrons moving toward, and backward from, the energy filter.

Those photoelectrons moving backward from the energy filter can be supplied by the electrons from the top contact instead of the bottom one because of the obstruction by the energy filter to the electrons from the bottom contact. Hence, the photoelectrons moving backward from the energy filter cause merely internal electron circulation and do not contribute to the measurable photocurrent. As a result, only the flux of photoelectrons moving toward the energy filter is responsible for the measured photocurrent. In the following analysis, only the number of photoelectrons moving toward the energy filter is considered.

During the transport in the second miniband, the photoelectrons may sequentially relax their energy and finally accumulate in the bottom state of the second miniband. This relaxation mechanism is called the intraminiband relaxation and results in the distribution for the number of photoelectrons moving toward the energy filter versus the second miniband energy, $N_{p}\left(E, P_{\text {in }}(\lambda)\right)$, as is schematically shown in the inset of Fig. 4, where $P_{\mathrm{in}}(\lambda)$ is the incident power of the photons with wavelength $\lambda$. The suffix indicates the $\psi_{N+1-p}^{1 \text { st }} \rightarrow \psi_{p}^{2 \text { nd }}$ transition as explained in Section IV-A.

In the meantime, it is also possible for the photoelectrons to be scattered into the high-energy state of the first miniband and to relax back into the bottom state of the first miniband. Such a process is called interminiband relaxation. When injecting into the energy filter after escaping the superlattice, the photoelectrons gain their kinetic energy through the electric field on the energy filter and also have the scattering probability which increases with their kinetic energy.

In an ideal case, the photoelectrons with energy $E$ may contribute to the carrier flux $J(E)$, which can be calculated from the electron wavefunction. Without taking the scattering in the energy filter into consideration, the transmission probability $T(E)$ of the photoelectrons through the blocking layer is easily estimated with WKB approximation. In reality, both the intraminiband and interminiband relaxations in the superlattice have to be considered, while the scattering effect in the energy filter is actually an intraband relaxation and has similar nature with the intraminiband relaxation of photoelectrons in the superlattice miniband. Therefore, in our model, this effect is included in the intraminiband relaxation in the superlattice. In summary, as indicated in Fig. 4, the whole mechanism of the voltage-dependent spectral responsivity consists of the light absorption in the superlattice region, the photoelectron relaxation during the transport in the second miniband, and the transmission through the energy filter. The scattering in the energy filter is included in the intraminiband relaxation for simplicity.

The aforesaid mechanisms will be discussed one by one in Section IV-A-IV-C, and then a complete equation for the voltage-tunable responsivity will be presented in Section IV-D.

\section{A. Absorption Coefficient}

Instead of considering the miniband in the 14-period superlattice as a continuous one, we calculate the measured quantity by counting the contributions from the 14 discrete resonant-tunneling states in the miniband. However, it is observed from Figs. 2 and 5 that neither the measured absorption spectrum nor the spectral responsivity shows the fine structure originated from the 14 discrete transitions. It is attributed that each discrete transition is broadened with an energy comparable to the one separating the neighboring states. In the following analysis, Lorentzian broadening is assumed in the modeling of the absorption spectrum. In addition, we also assume the spectral responsivity is broadened in the same fashion as the absorption spectrum for simplicity.

The transfer matrix method [11] was used to calculate the energies and corresponding wavefunctions of the resonant-tunneling states that form the two minibands in the superlattice region. According to the calculated oscillation strength, the electrons in the resonant-tunneling state $\psi_{N+1-p}^{1 s t}$ in the first miniband of the superlattice can only be excited to the corresponding resonant-tunneling state $\psi_{p}^{2 \text { nd }}$ in the second miniband 


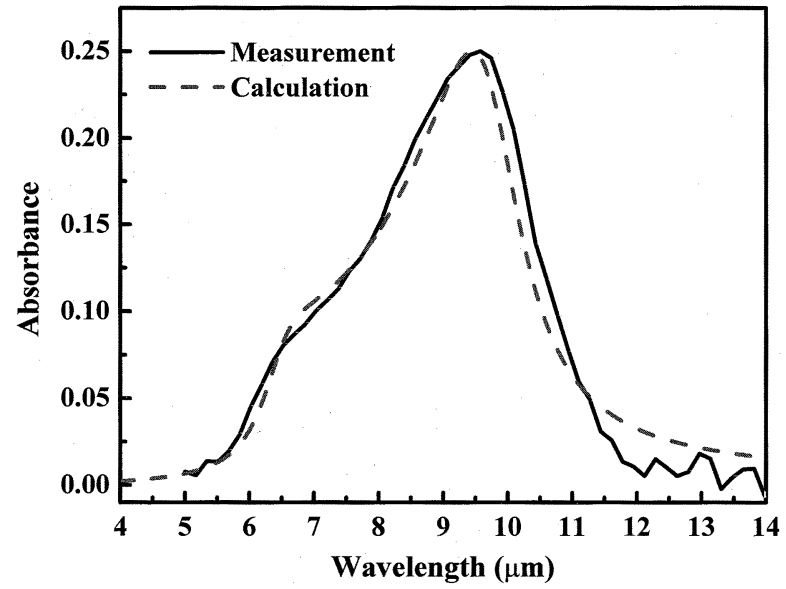

Fig. 5. Measured and calculated absorption spectrum for the TM-polarized light of the superlattice structure.

by absorbing infrared light. The overall absorption coefficient counting each individual transition from the first to the second miniband is given by [12], [13]

$$
\begin{aligned}
\alpha(\lambda)= & \sum_{p} \frac{e^{2} k T}{\varepsilon_{0} c n \hbar^{2} m^{*} \omega_{p} L}\left|\left\langle\psi_{p}^{2 \text { nd }}\left|p_{z}\right| \psi_{N+1-p}^{1 \text { st }}\right\rangle\right|^{2} \\
& \times \ln \left(\frac{1+\exp \left(\frac{\left[E_{f}-E_{N+1-p}^{\text {sst }}\right]}{K T}\right)}{1+\exp \left(\frac{\left[E_{f}-E_{p}^{2 \text { nd }}\right]}{K T}\right)}\right) \\
& \cdot \frac{\frac{\gamma}{\pi}}{\left(E_{p}^{2 \text { nd }}-E_{N+1-p}^{1 \text { st }}-\frac{h c}{\lambda}\right)^{2}+\gamma^{2}} \\
= & \sum_{p} \bar{\alpha}_{p}(\lambda)
\end{aligned}
$$

where the last multiplication factor in the summation is attributed to Lorentzian broadening and $\bar{\alpha}_{p}(\lambda)$ is the function defined in the summation and represents the absorption from the $\psi_{N+1-p}^{1 \text { st }} \rightarrow \psi_{p}^{2 \text { nd }}$ transition. From the curve fitting of the absorption spectrum, the superlattice parameters can be extracted. The substantial well and barrier widths are, in fact, 6.1 and $4.4 \mathrm{~nm}$, respectively, and the first and second miniband ranges are $53-62 \mathrm{meV}$ and $188-254 \mathrm{meV}$. These parameters will be utilized in the subsequent analysis of the voltage-dependent spectral responsivity. Fig. 5 shows the measured absorption spectrum, along with the fitting results.

\section{B. Carrier Flux and Transmission Probability}

One photoelectron represented by wavefunction $\psi$ can generate carrier flux $J$ given by [14]

$$
J=\frac{\hbar}{2 m i}\left(\psi^{*} \nabla \psi-\psi \nabla \psi^{*}\right)
$$

Fig. 6(a) shows the carrier flux of the resonant-tunneling states in the second miniband multiplied by the area of the mesa. The carrier flux shows a maximum near the middle of the second miniband corresponding to the excitation wavelength of $7.6 \mu \mathrm{m}$
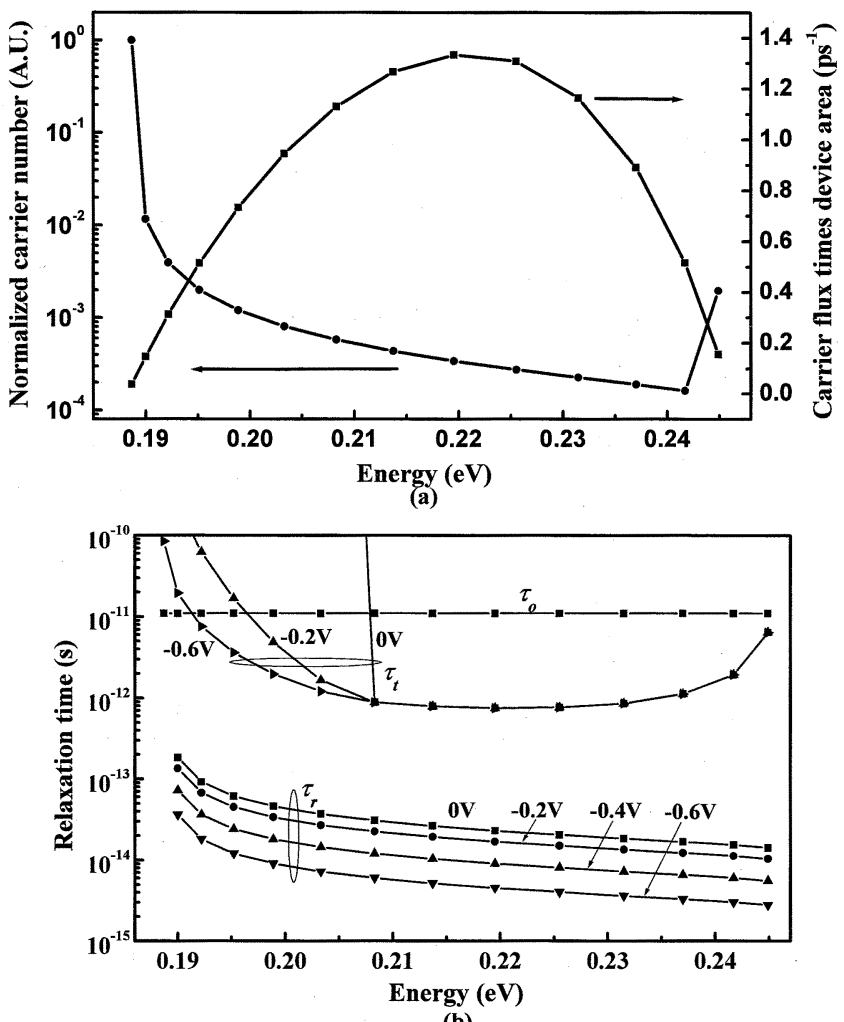

(b)

Fig. 6. (a) Carrier flux and normalized photoelectron number induced by $6.7-\mu \mathrm{m}$ light at $0 \mathrm{~V}$. The carrier flux is maximum at around the middle of the second miniband and is minimum in the bottom state of the second miniband. Most of the photoelectrons accumulate in the bottom state of the second miniband due to the fast intraminiband relaxation. (b) Simulation parameters of intraminiband and interminiband relaxation time constants along with carrier escaping time at several biases.

and the maximum value is 35 (5.8) times larger than the bottom (top) of the second miniband. The flux peak at $7.6 \mu \mathrm{m}$ does not appear in the experimental data of the responsivity. It is attributed to the relaxation of photoelectrons, which will be discussed in Section IV-E.

The carrier flux is partially reflected by the energy filter and partially passes through it. The associated transmission probability through the blocking layer can be calculated by using the WKB approximation and depends on the carrier energy and the electric field applied on the blocking layer. It can be written as in (3), shown at the bottom of the next page, where $E_{i}$ is the photoelectron energy, $F$ is the electric field applied on the energy filter, and $L_{b}$ and $E_{b}$ are the length and barrier height of the energy filter, respectively.

\section{Photoelectron Number Generated by Incident Light}

The number distribution for the photoelectrons toward the energy filter is actually time-dependent and denoted by $N_{p}\left(E_{i}, P_{\text {in }}(\lambda), t\right)$. The associated steady-state value is denoted by $N_{p}\left(E_{i}, P_{\text {in }}(\lambda)\right)$, as defined before. It is due to the photoelectrons generated by the $\psi_{N+1-p}^{1 \mathrm{st}} \rightarrow \psi_{p}^{2 \text { nd }}$ transition with incident power $P_{\text {in }}(\lambda)$ and those relaxed from higher energy states than $E_{i}$. For the incident power, the photoelectron 
number at energy $E_{p}$ in the second miniband is increased by the transition rate

$$
W_{p}(\lambda)=\frac{P_{\text {in }}(\lambda) \cdot\left(\bar{\alpha}_{p}(\lambda) L\right)}{\frac{2 h c}{\lambda}}
$$

where $\bar{\alpha}_{p}(\lambda)$ is the absorption coefficient defined in (1). The factor 2 explains that only half of the photoelectrons can travel toward the blocking layer and contribute to the responsivity. The excited photoelectrons suffer from both intraminiband and interminiband relaxations that are related to various scattering mechanisms such as phonon scattering and impurity scattering. The relaxation of photoelectrons between states only in the second miniband is attributed to the intraminiband relaxation, while relaxation from the second to the first miniband is the interminiband relaxation as schematically shown in Fig. 4. To find the photoelectron number in the second miniband generated by incident light, the rate equation of each resonant-tunneling state in the second miniband is analyzed. For simplicity, in the following analysis, the momentum perpendicular to the growth direction is neglected. Consider the photoelectron number as a result of the $\psi_{N+1-p}^{1 \text { st }} \rightarrow \psi_{p}^{2 \text { nd }}$ transition. For the incident optical power $P_{\text {in }}(\lambda)$ with photon energy $\hbar \omega$, the rate equation at energy $E_{i}$ in the second miniband is given by

$$
\begin{gathered}
\frac{\partial N_{p}\left(E_{i}, P_{\text {in }}(\lambda), t\right)}{\partial t}=G_{p}\left(E_{i}, P_{\text {in }}(\lambda), t\right)-\frac{N_{p}\left(E_{i}, P_{\text {in }}(\lambda), t\right)}{\tau_{r}\left(E_{i}\right)} \\
-\frac{N_{p}\left(E_{i}, P_{\text {in }}(\lambda), t\right)}{\tau_{o}\left(E_{i}\right)}-N_{p}\left(E_{j}, P_{\text {in }}(\lambda), t\right) \cdot J\left(E_{i}\right) \cdot A
\end{gathered}
$$

where $G_{p}\left(E_{i}, P_{\text {in }}(\lambda), t\right)$ is the photoelectron generation rate, $\tau_{r}\left(E_{i}\right)$ and $\tau_{o}\left(E_{i}\right)$ are the intraminiband and interminiband relaxation time, respectively, and $A$ is the mesa area of the detector. It should be noted that the scattering in the energy filter, which is expected to increase with the applied bias, is included in the parameter $\tau_{r}$, since the intraband relaxation in the energy filter has similar nature with the intraminiband relaxation. Therefore, the intraminiband relaxation $\tau_{r}$ is actually bias-dependent and expected to decrease with bias. The last term in (5) represents the total loss due to the carrier flux $J\left(E_{i}\right)$. It should be noted that no matter whether $E_{i}>E_{b}$ or $E_{i}<E_{b}$, the carrier flux of the photoelectron always represents a loss for $N_{p}$, be- cause the photoelectrons either leave the superlattice or change direction after they are reflected by the energy filter.

The steady-state solution for $N_{p}$ in (5) can be written as

$$
\begin{aligned}
N_{p}\left(E_{i},\right. & \left.P_{\text {in }}(\lambda)\right) \\
& =\frac{1}{\frac{1}{\tau_{r}\left(E_{i}\right)}+\frac{1}{\tau_{o}\left(E_{i}\right)}+J\left(E_{i}\right) \cdot A} G_{p}\left(E_{i}, P_{\text {in }}(\lambda)\right) .
\end{aligned}
$$

In the following, we will find an expression for the generation rate in (6). For the $E_{p}$ state, which the carriers are excited into by photons with energy $\hbar \omega$ through the $\psi_{N+1-p}^{1 \text { st }} \rightarrow \psi_{p}^{2 \text { nd }}$ transition, the generation rate $G_{p}\left(E_{p}, P_{\text {in }}(\lambda)\right)\left(E_{i}=E_{p}\right)$ is equal to the transition rate $W_{p}(\lambda)$ in (4). For each $E_{i}$ state with energy lower than $E_{p}$, the generation rate $G_{p}\left(E_{i}, P_{\text {in }}(\lambda)\right)$ comes from the intraminiband relaxation from the states with energy between $E_{i}$ and $E_{p}$. In addition to the intraminiband relaxation time, the function $D_{j}\left(E_{i}\right)$ is introduced for the intraminiband relaxation as the probability of carriers relaxed from energy state $E_{j}$ into a lower energy state $E_{i}$ in the second miniband. Therefore, the generation rate can be written with $D_{j}\left(E_{i}\right)$ as in (7), shown at the bottom of the page.Therefore, the steady-state light-induced carrier number $N_{p}\left(E_{i}, P_{\text {in }}(\lambda)\right)$ can be found by solving (6) and (7).

\section{Voltage-Dependent Spectral Responsivity}

The spectral responsivity is defined as the photocurrent divided by the incident power. Because of the intraminiband relaxation, the photocurrent may be due to the photoelectrons with energy $E_{i} \leq E_{p}$. Therefore, the responsivity can be written as

$$
\begin{aligned}
\bar{R}_{p}\left(\lambda, V_{a}\right) & \\
& =\sum_{i=1}^{p} e N_{p}\left(E_{i}, P_{\mathrm{in}}(\lambda)\right) \cdot J\left(E_{i}\right) \cdot A \cdot T \frac{\left(E_{i}, \frac{V_{a}}{L_{b}}\right)}{P_{\mathrm{in}}(\lambda)} .
\end{aligned}
$$

Because almost all of the external bias is dropped on the energy filter, the electric field $F$ on the energy filter may be written as $V_{a} / L_{b}$, where $L_{b}$ is the length of the energy filter. In order to compare the relaxation rate with the escaping carrier flux, the voltage-dependent escaping time constant is defined as

$$
\tau_{t}\left(E_{i}\right)=\left[J\left(E_{i}\right) \cdot A \cdot T\left(E_{i}, \frac{V_{a}}{L_{b}}\right)\right]^{-1}
$$

$$
\begin{cases}T\left(E_{i}, F\right)=1, & \text { for } E_{b}<E_{i} \\ T\left(E_{i}, F\right)=\exp \left[\frac{-4}{3 e F} \sqrt{\frac{2 m^{*}}{\hbar^{2}}}\left(E_{b}-E_{i}\right)^{3 / 2}\right], & \text { for } E_{b}-e F L_{b} \leq E_{i}<E_{b} \\ T\left(E_{i}, F\right)=\exp \left[\frac{-4}{3 e F} \sqrt{\frac{2 m^{*}}{\hbar^{2}}}\left[\left(E_{b}-E_{i}\right)^{3 / 2}-\left(E_{b}-E_{i}-e F L_{b}\right)^{3 / 2}\right]\right], & \text { for } E_{i}<E_{b}-e F L_{b}\end{cases}
$$

$$
\left\{\begin{array}{ll}
G_{p}\left(E_{p}, P_{\mathrm{in}}(\lambda)\right)=W_{p}\left(P_{\mathrm{in}}(\lambda)\right)=\frac{P_{\mathrm{in}}(\lambda) \cdot\left(\bar{\alpha}_{p}(\lambda) L\right)}{2 h \omega_{p}}, & E_{i}=E_{p} \\
G_{p}\left(E_{i}, P_{\mathrm{in}}(\lambda)\right)=\sum_{j=i+1}^{p} D_{j}\left(E_{i}\right) \frac{N_{p}\left(E_{j}, P_{\mathrm{in}}(\lambda)\right)}{\tau_{r}\left(E_{j}\right)}, & E_{i}<E_{p}
\end{array} .\right.
$$


which represents the time for the photoelectrons to escape the entire superlattice and tunnel through the blocking layer.

It is noted $\bar{R}_{p}\left(\lambda, V_{a}\right)$ in (8) is obtained by considering only the $\psi_{N+1-p}^{1 \text { st }} \rightarrow \psi_{p}^{2 \text { nd }}$ transition. The overall voltage-dependent spectral responsivity can be expressed as

$$
R\left(\lambda, V_{a}\right)=\sum_{p=1}^{N} \bar{R}_{p}\left(\lambda, V_{a}\right)
$$

where $N$ is the number of resonant-tunneling states in each miniband. Here, we are going to make several further assumptions in the calculation of (10). First, since for a high-energy state in the second miniband, there are more states with lower energy into which the photoelectrons can be relaxed, the intraminiband relaxation rate from the state $E_{i}$ is therefore assumed to be proportional to the number of the states with energy lower than $E_{i}$. That is, the intraminiband relaxation time constant is assumed to be $\tau_{r}\left(E_{i}\right)=\beta \cdot(i-1)^{-1}$ and $\beta$ is voltage dependent to include the scattering effect in the blocking layer. Second, the probability $D_{j}\left(E_{i}\right)$ is supposed to be uniformly distributed for the state with energy $E_{i}$ that is lower than $E_{j}$, i.e., $D_{j}\left(E_{i}\right)=1 /(j-1)$. Third, $\tau_{o}\left(E_{i}\right)$ is assumed to be independent of $E_{i}$ for simplicity. In this model, the carrier flux and tunneling probability are calculated values and the interminiband and intraminiband relaxation times are fitting parameters. In addition, the barrier height of the energy filter is also treated as a fitting parameter, since it is grown inaccurately. The calculated results of (10) for the voltage-dependent spectral responsivity are shown by the dashed curves in Fig. 2. The extracted barrier height is $208 \mathrm{meV}$ from the conduction band-edge of GaAs. Shown in Fig. 6(b) are the voltage-dependent intraminiband relaxation time along with the interminiband one and the escaping time under several bias voltages. Fig. 6(a) shows a representative normalized carrier distribution versus resonant-tunneling state energy in the second miniband at zero bias induced by the illumination of the short-wavelength $(6.7 \mu \mathrm{m})$ light. The agreement between the experimental and simulated spectral responsivity offers some insight into the photoelectron transport and relaxation mechanisms, which will be discussed next.

\section{E. Discussion}

As expected, the photoelectron number distribution $N_{p}$ in Fig. 6(a) shows a peak at the state $E_{p}$, into which the electrons are excited by photons. However, due to the intraminiband relaxation, almost $97.7 \%$ of the total photoelectrons are relaxed and accumulated in the bottom state of the second miniband. The extracted interminiband relaxation time from the fitting is $11 \mathrm{ps}$ for our sample. This value is consistent with the intersubband relaxation time in the quantum-well infrared photodetectors which is in the range from several picoseconds to tens of picoseconds [14]-[18].

The escaping time for the photoelectrons under several bias voltages is shown in Fig. 6(b). It is noted from (3) that under zero bias, the transmission probability approaches zero if the photoelectron energy is lower than the energy filter, while the transmission probability equals 1 if the photoelectron energy is higher than the energy filter. This causes the steep increment of escaping time below the barrier height $208 \mathrm{meV}$ of the energy filter under zero bias. The fitting barrier height corresponding to the aluminum fraction $x=0.256$ is actually higher than the designed value of $186 \mathrm{meV}(x=0.23)$. The conduction band offset for $\mathrm{Al}_{x} \mathrm{Ga}_{1-x} \mathrm{As}$ is taken as $810 x \mathrm{meV}^{7}$. In the growth of the sample, the $\mathrm{Al}$ sources for our superlattice barrier and energy filter were different. The superlattice barriers are grown accurately but the energy filter seems not. It is our belief that the fitting parameter is more accurate than the designed one since the applied bias must be as high as $-0.6 \mathrm{~V}$ to extract the accumulated carriers in the bottom state of the second miniband, as shown in Fig. 3.

The extracted intraminiband relaxation time is much shorter than the interminiband one and varies from several tenths of picoseconds to several femtoseconds for the bias between 0 and $-0.6 \mathrm{~V}$, as shown in Fig. 6(b). The intraminiband relaxation rate increases with the second miniband energy since, for a high-energy state in the second miniband, there are more states with energy lower than it, into which the photoelectrons can be relaxed into. Besides, the aggravation of intraminiband relaxation rate with applied voltage is attributed to the scattering in the energy filter. The fast intraminiband relaxation compared with the interminiband one is consistent with the fact that the spectral responsivity does not show a maximum at the wavelength $7.6 \mu \mathrm{m}$, corresponding to the maximum of carrier flux which is 35 times lager than that in the bottom of the second miniband. It is because only a small fraction of the photoelectrons can stay in the high-energy states of the second miniband while most of them are accumulated in the bottom state of the second miniband by sequential intraminiband relaxation. The detailed physical mechanisms about the relaxation time would be complicated and will be studied in the future.

Fig. 3 shows the measured and calculated responsivity versus bias voltage at 6.7 and $9.7 \mu \mathrm{m}$. The data for the responsivity at $6.7 \mu \mathrm{m}$ reveals some interesting results due to the relaxation of the photoelectrons. Here we discuss the responsivity at $6.7 \mu \mathrm{m}$ in more detail. The open squares and circles shown in Fig. 3 are the calculated data corresponding to the responsivity caused by all the excited photoelectrons in the second miniband and by the photoelectrons with energy higher than the energy filter, respectively. Due to the small tunneling probability at low bias, most of the photoelectrons accumulated in the bottom state of the second miniband can not contribute significantly to the responsivity. As can be observed from the proximity of the open squares and circles at low biases in Fig. 3, the responsivity is primary dominated by the emission photocurrent, which is caused by the photoelectrons with energy higher than the energy filter. Although the photoelectrons with energy higher than the energy filter are much smaller in number than those accumulated in the bottom state of the second miniband, due to the larger carrier flux than the smallest one of the bottom state as shown in Fig. 6(a), they can generate $8 \mathrm{~mA} / \mathrm{W}$ of the responsivity at zero bias.

Even though more photoelectrons accumulated in the bottom state of the second miniband can be collected under high bias, they only generate $32 \mathrm{~mA} / \mathrm{W}$ at $-0.6 \mathrm{~V}$, which is four time larger than the responsivity at zero bias. It is because of the smallest carrier flux of the bottom state in the second miniband. This indicates that the responsivity can be enhanced by reducing the 
intraminiband relaxation to allow more photoelectrons to stay in the state with larger carrier flux. Also noted in Fig. 3 is the responsivity caused by the emission photocurrent as represented by the open circles reduces with increasing bias because of the increasing intraminiband relaxation as shown in Fig. 6(b). At $-0.6 \mathrm{~V}$, the responsivity at $6.7 \mu \mathrm{m}$ is dominated by the tunneling photocurrent, which is the same as the responsivity at $9.7 \mu \mathrm{m}$. Since the tunneling probability and the carrier flux are the same, the responsivity at $9.7 \mu \mathrm{m}$ is 3.4 times the one at $6.7 \mu \mathrm{m}$ because of the larger transition rate for the 9.7- $\mu \mathrm{m}$ light. In brief, the photocurrent model provides a detailed explanation for the voltage dependent responsivity, including the nonzero responsivity at zero bias at short wavelength, the increment of short-wavelength responsivity with the magnitude of bias, and the responsivity at long wavelengths, which is mainly tunneling photocurrent.

From the discussion above, it was found that the much faster intraminiband relaxation is the dominant factor in limiting the maximum achievable responsivity of the superlattice infrared photodetector. However, the large ratio of the interminiband time constant over the intraminiband one suggests that the superlattice structure can be utilized in the design of quantum-cascade lasers to achieve population inversion [20], [21]. Nevertheless, the tunable spectral responsivity exhibits the potentiality in versatile applications, including temperature sensing and target discrimination.

\section{SUMMARY}

We have designed a superlattice with an energy filter to investigate the photoelectron transport in the superlattice miniband. In addition, this structure can be applicable as an infrared photodetector, which shows the advantages of lower oprating voltage $(0 \sim-0.7 \mathrm{~V})$, wider and tunable spectral responsivity, and flexible miniband engineering. A model based on the rate equation is suggested for the measured voltage-dependent behavior of the photoresponse. From this model, it is found that the intraminiband relaxation process (subpicosecond) is much faster than both the interminiband relaxation $(11 \mathrm{ps})$ and the carrier escaping time (with a minimum of $0.75 \mathrm{ps}$ ) and is the dominant mechanism for the responsivity. This indicates that the responsivity can be increased by the reduction of fast intraminiband relaxation. Therefore, this model not only provides an insight into the transport of the photoelectrons in the superlattice with an energy filter, but also a tool to further improve the detector performance.

\section{REFERENCES}

[1] K. K. Choi, The Physics of Quantum Well Infrared Photodetectors, Singapore: World Scientific, 1997, ch. 8.

[2] A. Kastalsky, T. Duffield, S. J. Allen, and J. Harbison, "Photovoltaic detection of infrared light in a GaAs/AlGaAs superlattice," Appl. Phys. Lett., vol. 52, pp. 1320-1322, Apr. 1988.

[3] O. Byungsung, J.-W. Choe, M. H. Francombe, K. M. S. V. Bandara, and D. D. Coon, "Long-wavelength infrared detection in a Kastalsky-type superlattice structure," Appl. Phys. Lett., vol. 57, pp. 503-505, July 1990.

[4] S. D. Gunapala, B. F. Levine, and N. Chand, "Bound to continuum superlattice miniband long wavelength $\mathrm{GaAs} / \mathrm{Al}_{x} \mathrm{Ga}_{1-x}$ As photoconductors," J. Appl. Phys., vol. 70, pp. 305-308, July 1991.

[5] K. M. S. V. Bandara, J.-W. Choe, and M. H. Francombe, "GaAs/AlGaAs superlattice miniband detector with $14.5 \mu \mathrm{m}$ peak response," Appl. Phys. Lett., vol. 60, pp. 3022-3024, June 1992.
[6] S. Gunapala, S. Bandara, J. Bock, M. Ressler, J. Liu, J. Mumolo, S. Rafol, D. Ting, and M. Werner, "Large format long-wavelength GaAs/AlGaAs multi-quantum well infrared detector detector arrays for astronomy," in Proc. SPIE, vol. 4288, 2001, pp. 278-285.

[7] C. C. Chen, H. C. Chen, M. C. Hsu, W. H. Hsieh, and C. H. Kuan, "Performance and application of a superlattice infrared photodetector with a blocking barrier," J. Appl. Phys., vol. 91, pp. 943-948, February 2002.

[8] H. C. Liu and F. Capasso, "Quantum well infrared photodetector physics and novel devices," in Intersubband Trasitions in Quantum Wells-Physics and Device Applications I. London, U.K.: Academic, 2000, pp. 135-136.

[9] B. K. Ridley, Quantum Processes in Semiconductors. Oxford, U.K.: Oxford Univ. Press, 1993, ch. 4.

[10] J. Singh, Physics of Semiconductors and Their Heterostructures. New York: McGraw-Hill, 1993, ch. 11

[11] S. L. Chang, Physics of Optoelectronic Devices. New York: Wiley, 1995 , ch. 4.

[12] M. Helm, W. Hilber, and T. Fromherz, "Infrared absorption in superlattices: A probe of the miniband dispersion and the structure of the impurity band," Phys. Rev. B, vol. 48, pp. 1601-1606, July 1993.

[13] S. Nojima, "Intraband optical absorption in semiconductor superlattices," Phys. Rev. B, vol. 41, pp. 10214-10 217, May 1990.

[14] K. L. Wang and P.-F. Yuh, "Theory and applications of band-aligned superlattices," IEEE J. Quantum Electron., vol. 25, pp. 12-19, Jan. 1989.

[15] J. A. Brum and G. Bastard, "Resonant carrier capture by semiconductor quantum wells," Phys. Rev. B, vol. 33, pp. 1420-1423, Jan. 1986.

[16] B. F. Levine, A. Zussman, S. D. Gunapala, M. T. Asom, J. M. Kuo, and W. S. Hobson, "Photoexcited escape probability, optical gain and noise in quantum well infrared photodetectors," J. Appl. Phys., vol. 72, pp. 4429-4443, Nov. 1992.

[17] K. K. Choi, C. Y. Lee, M. Z. Tidrow, and W. H. Chang, "Performance assessment of quantum well infrared photodetectors," Appl. Phys. Lett., vol. 65, pp. 1703-1705, Sept. 1994.

[18] E. Rosencher, F. Luc, P. Bois, J. Nagle, and Y. Cordier, "Capture time versus barrier thickness in quantum-well structures measured by infrared photoconductive gain," Appl. Phys. Lett., vol. 63, pp. 3312-3314, Dec. 1993.

[19] E. Rosencher, B. Vinter, F. Luc, L. Thibaudeau, P. Bois, and J. Nagle, "Emission and capture of electrons in multiquantum-well structures," IEEE J. Quantum Electron., vol. 30, pp. 2875-2888, 1994.

[20] F. Capasso, A. Tredicucci, C. Gmachl, D. L. Sivco, A. L. Hutchinson, A. Y. Cho, and G. Scamarcio, "High-performance superlattice quantum cascade lasers," IEEE J. Select. Topics Quantum Electron., vol. 5, pp. 792-807, May 1999.

[21] A. Tredicucci, F. Capasso, C. Gmachl, D. L. Sivco, A. L. Hutchinson, and A. Y. Cho, "High-performance quantum cascade lasers with electricfield-free undoped superlattice," IEEE Photon. Technol. Lett., vol. 12, pp. 260-262, Mar. 2000.

Chun-Chi Chen was born in Taiwan, R.O.C. He received the Ph.D. degree in electrical engineering from National Taiwan University, Taipei, Taiwan, R.O.C., in 2002. His studies focused on the design and analysis of multicolor superlattice infrared photodetectors.

$\mathrm{He}$ is currently with the Research and Development Department of Taiwan Crystal Corporation, Taiwan, R.O.C., involved in developing high-precision oscillators.

H. C. Chen was born in Tainan, Taiwan, in 1977. He received the B.S. and M.S. degrees in electrical engineering from National Taiwan University, Taiwan, R.O.C., in 1999 and 2001, respectively.

Since 2001, he has been with Media Tek Inc., Taiwan, R.O.C. His research interests include infrared photo-detectors, coding theory, optical-storage systems, and VLSI architecture.

Mao-Chieh Hsu was born in Taipei, Taiwan, R.O.C. He received the M.S. degree in electrical engineering in physics from National Taiwan University, Taipei, Taiwan, in 2000. His Ph.D. studies focus on the design and characterization of infrared devices using the intersubband transitions in quantum well or superlattice structures.

He currently serves in the Research and Development Department, Copax Photonic Corporation, Taiwan, R.O.C., responsible for developing high-speed fiber communication receivers. 
Wen-Hsing Hsieh was born in Taipei, Taiwan, R.O.C. He received the M.S. degree in physics from National ChungHsing University, Taichung, Taiwan, R.O.C., in 1997, and is currently working toward the Ph.D. degree in electrical engineering at National Taiwan University, Taipei, Taiwan, R.O.C.

His current research interests include the high-frequency magnetotransport properties of a two-dimensional electron system and spin obital effects in quantum dots or quantum-point contacts.

Chieh-Hsiung Kuan (M'95) was born in Taipei, Taiwan, R.O.C., in 1962. He received the B.S. degree in electrical engineering from National Taiwan University, Taipei, Taiwan, R.O.C., in 1985, and the M.S.A. and Ph.D. degrees in electrical engineering from Princeton University, Princeton, NJ, in 1990 and 1994, respectively. His Ph.D. work involved dark current and noise characteristics of the infrared hot-electron transistors, in cooperation with the U.S. Army Laboratory, Fort Monmouth, NJ.

In 1994, he joined the Department of Electrical Engineering, National Taiwan University, as an Associate Professor. His current research interests include infrared photodiodes for room-temperature operation, quantum-well infrared photodetectors and lasers, and topics on how to measure and suppress the noise in detectors.

Dr. Kuan is a member of Phi-Tau-Phi.

Shiang-Yu Wang was born in Taiwan, R.O.C., in 1972. He received the B.S. degree in physics from National Taiwan University, Taipei, Taiwan, R.O.C., in 1994 and the Ph.D. degree in electronic engineering from National Chiao Tung University, HsinChu, Taiwan, R.O.C., in 1999. His dissertation focused on quantum-well and quantum-dot infrared detectors.

He is an Assistant Research Fellow with the Institute of Astronomy and Astrophysics, Academia Sinica, Taiwan, R.O.C. His research interests include molecular-beam-epitaxy techniques, growth and characterization of quantum-well and self-organized quantum-dot infrared detectors, and infrared instrument development for large telescopes.

Dr. Wang is a member of Phi Tau Phi.
Chien-Ping Lee (M'80-SM'94-F'00) received the B.S. degree in physics from National Taiwan University, Taipei, Taiwan, R.O.C., in 1971 and the Ph.D. degree in Applied Physics from the California Institute of Technology, Pasadena, in 1978.

$\mathrm{He}$ was with Bell Laboratories and later Rockwell International until 1987. While at Rockwell, he was a Department Manager, responsible for developing high-speed semiconductor devices. He became a Professor at National Chiao-Tung University, Hsinchu, Taiwan, R.O.C., in 1987. He was also appointed Director of the Semiconductor Research Center and, later, the first Director of the National Nano Device Laboratory. Currently, he is the Director of the Nano Science and Technology Center, National Chiao Tung University. He is well recognized in the field of semiconductor research $\mathrm{He}$ is an expert in compound semiconductor devices and was the pioneer of the development of optoelectronic integrated circuits (OEIC), high electron mobility transistors (HEMTs), and ion-implanted MESFETs. His current interests include semiconductor nano structures, quantum devices, spintronics, and heterjunction bipolar transistors. He has graduated $20 \mathrm{Ph} . \mathrm{D}$. students and more than 40 Master's degree students.

Dr. Lee was the Founding Chair of the IEEE LEOS Taipei Chapter and has also served in the IEEE EDS Taipei Chapter. He has organized and served in several international conferences. He was awarded the Engineer of the Year Award from Rockwell in 1982, the Best Teacher Award from the Ministry of Education in 1993, the Outstanding Engineering Professor Award from the Chinese Institute of Engineers in 2000, the Outstanding Research Award from the National Science Council in 1993, 1995, and 1997, the Outstanding Scholar Award from the Foundation for the Advancement of Outstanding Scholarship in 2000, and the Academic Achievement Award from the Ministry of Education in 2001. 